\title{
Lingüística de corpus y fraseología contrastiva (alemán-español): Las combinaciones usuales de estructura [PREP + S]. El caso de entre lágrimas y unter Tränen
}

\author{
Corpus linguistics and contrastive phraseology (German-Spanish): \\ The multi word units of $[\mathrm{PREP}+\mathrm{N}]$ structure. \\ The case of entre lágrimas and under Tränen
}

\begin{abstract}
Ana Mansilla
Universidad de Murcia. anamansi@um.es

Recibido: 14/05/2017. Aceptado: 25/10/2017

Resumen: En el presente artículo analizamos la fijación externa e interna que presenta el binomio [entre $+S$ ] con artículo cero en español, y sus equivalentes en alemán en las combinaciones usuales entre lágrimas y unter Tränen. Los datos son extraídos del corpus DeReKo (Das Deutsche Referenzkorpus) y del Sketch Engine, deTenTen 13 y eseuTenTen11. En primer lugar, presentamos los objetivos del proyecto en el que este trabajo está enmarcado. En un segundo punto exponemos las diferentes aplicaciones de la lingüística de corpus en el ámbito de la fraseología. Por último, en base a los corpus consultados comentamos convergencias o divergencias más notables de las combinaciones usuales objeto de estudio.
\end{abstract}

Palabras clave: fraseología; lingüística de corpus; combinaciones usuales; alemán; español.

\begin{abstract}
The present article analyses the external and internal fixation of the binomial pattern [entre $+N N]$ with zero article in Spanish and its equivalents in German. In particular, the article focuses on the expressions entre lágrimas/unter Tränen. The data have been extracted from the DeReKo corpus (Das Deutsche Referenzkorpus) and from the Sketch Engine corpora deTenTen 13 and eseuTenTen 11. First, the article will present the objectives of the research project of which this study forms part. Then, I will address some applications of corpus linguistics in the field of phraseology. Finally, similarities and differences between the expressions investigated will be analysed based on the evidence obtained from the corpora.
\end{abstract}

Keywords: phraseology; corpus linguistics; common multi-word units; German; Spanish.

\) Mansilla, Ana. 2017. "Lingüística de corpus y fraseología contrastiva (alemán-español): Las combinaciones usuales de estructura [PREP + S]. El caso de entre lágrimas y unter Tränen". Quaderns de Filologia: Estudis Lingüístics 22: 147164. doi: 10.7203 qf.22.11306 



\section{Introducción}

Objeto del presente artículo es abordar los desarrollos recientes de la fraseología desde la perspectiva de la lingüística de corpus y analizar las posibilidades que ofrecen las tecnologías lingüísticas para la resolución de aspectos pendientes como el significado, la función o la forma de las unidades fraseológicas. Este terreno emergente de la lingüística de corpus en el ámbito de la fraseología bilingüe (alemán-español) se encuentra, a nuestro modo de ver, escasamente explorado, hecho que nos ha empujado a escribir este trabajo. Enfocamos nuestro estudio hacia las combinaciones usuales [PREP $+\mathrm{S}$ ] basándonos en la teoría de Kathrin Steyer (2013). En especial, prestamos atención a las combinaciones usuales [PREP $+\mathrm{S}$ ] entre lágrimas y su equivalente alemán unter Tränen. La finalidad es detectar convergencias y divergencias entre las distintas CU, comprobar los perfiles sintagmáticos, tanto la fijación externa (colocados sustantivos y verbales que funcionan de nodo del modificador preposicional), como la fijación interna (slots internos) en ambos sistemas fraseológicos y si es posible sistematizarlos semántica $\mathrm{y}$ fraseológicamente.

\section{Marco del Proyecto. Breve descripción}

Este trabajo se enmarca en el proyecto de investigación Combinaciones fraseológicas del alemán de estructura [PREP + S]: patrones sintagmáticos, descripción lexicográfica y correspondencias en español en curso del grupo FRASESPAL ${ }^{1}$ cuya finalidad es extraer, inventariar y describir las combinaciones usuales $(\mathrm{CU})$ de estructura $[\mathrm{PREP}+\mathrm{S}] \mathrm{de}$ diferentes corpus. Seguimos un método inductivo, es decir, empleamos las herramientas y los datos estadísticos que nos proporciona el corpus con el fin de extraer información que sería invisible en ausencia de

\footnotetext{
${ }^{1}$ Proyecto del Ministerio de Ciencia e Innovación (FFI2013-45769-P) con el título Combinaciones fraseológicas del alemán de estructura [PREP + S]: patrones sintagmáticos, descripción lexicográfica y correspondencias en español, promovido por el equipo de investigación FRASESPAL dirigido por Carmen Mellado Blanco de la Universidad de Santiago de Compostela con la colaboración de Kathrin Steyer, directora del proyecto "Usuelle Wortverbindungen" del IDS (http://www1.ids-mannheim.de/ lexik/uwv.html). Los resultados del proyecto serán publicados en la plataforma online OWID del IDS http://www.owid.de/wb/uwv/start.html.
} 
corpus, y que resulta relevante para nuestro estudio. En nuestro proyecto excluimos las CU que forman parte de "Funktionsverbgefüge" o complementos preposicionales regidos por verbos, sustantivos o adjetivos, (padecer de, tener que ver con, sucumbir a, etc.). Asimismo, manejamos herramientas diseñadas en el IDS (Institut für Deutsche Sprache) como el programa COSMAS II (Corpus Search, Management and Analysis System) y Lexpan (Lexical Patterns Analyzer) que en base a las listas de Kwics (Key Word in Context) facilita el análisis de los slots internos o Lückenfüller de las CU.

Asimismo, compilamos la información en base al corpus Sketch Engine, en concreto en base al corpus del alemán deTenTen 13 y los corpus European Spanish Web 2011 y eseuTenTen11 para el español.

Fundamentamos nuestro análisis a partir de dos parámetros: la fijación externa hace referencia a los colocados verbales y sustantivos que aparecen a la derecha y a la izquierda del nodo (cotexto anterior o posterior) o la palabra o palabras que se están estudiando, es decir, en contacto directo o no con el nodo $[\mathrm{X}(\ldots) \mathrm{PREP}+\mathrm{S}(\ldots) \mathrm{X}]$, con el fin de que se puedan constatar modelos recurrentes que tienden a la lexicalización (Mellado Blanco, 2015) y la fijación interna [PREP $+X+S$ ], esto es, qué tipo de slots internos aparecen en el discurso entre la preposición y el sustantivo: [con X dolores] (con fuertes dolores); [unter X Schmerzen] (unter starken Schmerzen).

Se nos antoja de obligada mención resaltar la función semántica de las preposiciones y de los sustantivos en las CU que estudiamos [PREP $+\mathrm{S}$ ] y si cada unidad léxica mantiene sus significados léxicos o no, esto es, si actúan con un sentido literal o con uno figurado, porque la interacción entre forma y significado influye directamente en la mayor o menor idiomaticidad y por tanto lexicalización de las CU.

Las combinaciones usuales objeto de nuestro estudio lo constituyen combinaciones de palabras que poseen constituyentes fijos y otros variables que, aunque son slots libres, están sujetos a ciertas restricciones semántico-combinatorias [unter Vorbehalt $X_{\text {dem Abkommen/einem Beitritt/ }}$ dem Projekt $z$ ustimmen], [con $X_{\text {tono/mueca/sonrisa }}$ de satisfacción]. Steyer delimita conceptualmente el término de combinación usual (usuelle Wortverbindung) como sigue:

Usuelle Wortverbindungen (UWV) sind als polylexikalische, habitualisierte sprachliche Zeichen zu verstehen, die spezifischen Beschränkungen unterliegen. Diese Beschränkungen können alle Ebenen der Spra- 
che betreffen. Sie ergeben sich aber primär nicht aus dem Sprachsystem, etwa bedingt durch transformationelle Defekte oder semantische Selektionsbeschränkungen, sondern aus dem rekurrenten Gebrauch dieser mehrgliedrigen Entitäten (Steyer, 2013: 16).

Desde un punto de vista semántico estas construcciones presentan un componente pragmático "adicional", no deducible de la suma de los significados parciales de sus constituyentes. Steyer (2013) sustenta su teoría en datos estadísticos del corpus y establece diferentes parámetros para valorar la mayor o menor fijación de las combinaciones usuales objeto de estudio:

- El grado de fijación de la preposición y del sustantivo.

- El grado de posible saturación entre preposición y sustantivo.

- La interacción entre forma y significado de las CU.

- La ausencia de determinante entre preposición y sustantivo.

La teoría de Steyer (2013) se sustenta a grandes rasgos en aquellos fraseologismos que se han situado durante mucho tiempo en la periferia de la fraseología alemana, y, por tanto, no han sido abordados de forma sistematizada y lo conforman los Muster, Schemata, Schablonen. Al respecto cabe citar el término de Modellbildung acuñado por Häusermann (1977:30), o el de Phraseoschablone que apunta Fleischer (1997: 130) y que hace referencia a esquemas que encierran una interpretación semántica fija y están sujetos a una especia de "idiomaticidad sintáctica". Algunos ejemplos que Fleischer cita en su obra se ajustan al esquema "X ist X" (p. ej. sicher ist sicher, Urlaub ist Urlaub, geschenkt ist geschenkt, etc.). En este sentido, Burger (2015: 45) y Dobrovol' skij (2011) abogan por el término Schema que hace referencia a un esquema sintáctico con una semántica irregular, cuyos slots son rellenados por componentes léxicos libres aunque sometidos a ciertas restricciones semánticas: $\left[\mathrm{PRON}_{\text {der/die/ }}\right.$ und $\left.\mathrm{X}_{\text {INFINITIVo }}\right]$ : der und singen!, der und diktieren [iél cantando, iél imponiéndose!], entre otros.

\section{Lingüística de corpus}

El concepto lingüística de corpus está indisolublemente asociado con la esfera de la lingüística computacional (o lingüística informática) que, según Chantal Pérez \& Antonio Moreno (2009: 68), constituye "un 
campo científico de carácter interdisciplinar, vinculado a la lingüística y a la informática, cuyo fin fundamental es la elaboración de modelos computacionales que reproduzcan distintos aspectos del lenguaje humano y que faciliten el tratamiento informatizado de las lenguas".

La irrupción de las nuevas tecnologías ha enriquecido sobremanera la visión de conjunto del fenómeno de la lingüística. Entre las múltiples aplicaciones de la lingüística de corpus, hay que señalar la frecuencia de palabras en torno a un campo semántico determinado, la elaboración de modelos lingüísticos (gramática sintagmática generalizada), o la descripción de diferentes niveles de la lengua (sintaxis, semántica, pragmática). De igual modo, cabe mencionar el campo de las tecnologías del habla (reconocimiento del habla, la síntesis del habla) o la traducción automática, o asistida por ordenador, entre otros. Asimismo, se puede aplicar al ámbito de la fraseología cuando de lo que se trata es del hábito colocacional de las palabras, esto es, de la frecuencia de coaparición (aparición simultánea) de varias palabras. Se pueden extraer y ordenar frecuencias por orden alfabético, o índices estadísticos de palabras que aparezcan a la derecha y a la izquierda del nodo, entre otros.

Sin dejar de lado la corriente computacional, es justo mencionar la labor desempeñada por Sinclair (1991: 170)², para quien es sustancial la frecuencia de coaparición de las unidades que integran la colocación o la combinación de palabras, acogiéndose al idiomatic principle. En relación a este principio, los hablantes suelen emplear unas palabras con una frecuencia mayor que otras, lo que da lugar a combinaciones "léxicas semiprefabricadas". Asimismo, las coocurrencias léxicas pueden dar cuenta del perfil colocacional, los patrones coligacionales o las preferencias semánticas.

En el ámbito de la lexicografía los corpus electrónicos se han vuelto prácticamente imprescindibles al aportar información relevante desde un punto de vista pragmático (Sánchez \& Almela, 2010: 5), porque el significado de una palabra es su uso en un contexto, una idea que ya en su día formuló Wittgenstein en su obra Philosophische Untersuchungen (1953) hace más de seis décadas: "Die Bedeutung eines Wortes ist sein Gebrauch in der Sprache".

\footnotetext{
${ }^{2}$ Sinclair hace referencia al node (núcleo), a los collocates (colocados) y span (espacio de texto) para entender con más claridad qué se entiende por colocación, siendo especialmente relevante el espacio que dista entre dos o más palabras.
} 


\section{Análisis de la CU con estructura [UNTER + S]/[ENTRE + S]. El caso unter Tränen y entre lágrimas}

A partir del rastreo en el corpus DeTenTen y EnTenTen de Sketch Engine queremos mostrar la combinatoria del cotexto de las combinaciones usuales entre lágrimas y su equivalente en alemán unter Tränen. La búsqueda que llevamos a cabo tiene en cuenta si la $\mathrm{CU}$ aparece al principio o no de la frase. Hemos observado que la frecuencia de aparición de las CU alemanas es mayor que las españolas, y está relacionado con aspectos sintácticos intrínsecos a cada lengua (unter Tränen, 10299 frente a entre lágrimas 710 ocurrencias).

Como hemos señalado más arriba, en nuestro proyecto incidimos tanto en la fijación externa [X entre lágrimas X] como en la fijación interna [entre $\mathrm{X}$ lágrimas], cuyo slot $\mathrm{X}$ suele estar saturado por adjetivos. La alta frecuencia de aparición de determinados colocados verbales o sustantivos puede desembocar en patrones o construcciones sintácticas que contienen un significado más o menos idiomático (Phraseoschablonen).

De las cinco acepciones que el DRAE recoge para el lexema lágri$m a$, la primera es la que nos interesa para nuestro estudio: (1) "Cada una de las gotas que segrega la glándula lagrimal (usado más en plural)". Por su parte, el Deutsches Wörterbuch der deutschen Sprache (DWDS) registra una acepción para el lexema Träne: (1) "von den Tränendrüsen im Auge abgesonderte, klare Flüssigkeit".

La preposición unter en la CU unter Schmerzen designa un "Begleitumstand" (Helbig \& Buscha, 1996: 439), esto es, una circunstancia (valor modal) que va de la mano de la acción principal. A modo de ejemplo, sirvan de ilustración los siguientes ejemplos extraídos de Helbig \& Buscha (1996: 439):

(1) Unter großem Beifall wurde der Redner vorgestellt.

(2) Unter Jubel und Gelächter fiel der Vorhang.

A este respecto, procede mencionar la publicación de Tibor Kiss (2014) que, de forma pormenorizada, aborda las preposiciones desde diferentes enfoques (sintáctico, pragmático o semántico). En lo que a la preposición unter se refiere, de las once acepciones que presentan los 
autores la tercera es la que mejor se ajusta al significado de la preposición unter en la CU unter Tränen (3b. Begleitumstand/2 Vorgänge):

Begleitumstände umfassen sowohl äußere Begleitumstände (wie Beifall) als auch Gefühle und Gemütszustände, die eine Handlung begleiten. Die Bedeutung tritt nur bei Modifikation von Ereignissen, Handlungen oder Zuständen auf. Die Lesart wird häufig durch unbelebte oder fehlende Agenten hervorgerufen. In der Semantik sind zwei identifizierbare Vorgänge angelegt, von denen der eine den anderen begleitet. Bei dem zweiten Vorgang handelt es sich um die Umstände der Handlung, die oftmals nicht intentional verursacht werden (Kiss et al., 2014: 188).

En lo que concierne al español, la preposición entre especifica un significado locativo que etimológicamente procede de inter 'en el interior de algo discontinuo' (Martínez García, 2012: 25) y que, a su vez, proviene de la locución prepositiva intro usque (hasta dentro de). En latín clásico la preposición de acusativo inter designaba tanto un valor locativo (inter multitudinem 'en medio de la multitud') como uno temporal (inter noctem 'durante la noche'). En opinión de Cabezas Holgado (2013: 17), las propiedades léxicas del núcleo predicativo entre se resumen en dos: valor locativo y valor colectivo.

Existe un grupo numeroso de $\mathrm{CU}$ que subyacen al esquema siguiente [unter/entre $\mathrm{S}_{\text {plural /sentimientos (movimientos corporales, gritos, partes del discurso }}$ por ejemplo, entre protestas - unter Protesten; entre gemidos - unter Seufzern; entre lágrimas - unter Tränen; entre risas - unter Gelächter, etc. Los sustantivos que ocupan el slot $\mathrm{S}$ lo conforman semánticamente sustantivos del ámbito de la comunicación verbal o de la expresión corporal (Mellado Blanco \& López Meirama en prensa).

Por lo que se refiere a la preposición entre en la CU entre lágrimas aquella expresa dos valores: temporal de simultaneidad y modal. Un estado se desarrolla de manera paralela a la acción que expresa el verbo principal. El hecho de que se produzcan dos eventos de forma simultánea radica en la naturaleza semántica de los sustantivos que acompañan a la preposición, por ser en su mayoría de tipo deverbal (entre llantos, sollozos, risas, etc.). Partiendo en este caso del significado del sustantivo podemos, en cierto modo, llegar al significado real de la preposición: 
(3) Unter Tränen sagt er in einem Interview: "Ich wollte immer nur ein ganz normaler Junge sein. Aber das Schicksal hat es anders gewollt. [http://casting.mattschiibe.ch/2009/06/]

Como se desprende del ejemplo (3), la CU unter Tränen suele tener como equivalencia un gerundio en español (unter Tränen sagt er-dice llorando). Si bien existen sustantivos con sufijos sustantivadores que, desde un punto de vista morfológico, evidencian su relación con los sustantivos, se les incluye en el grupo de los sustantivos deverbales, aún cuando no describan sensu stricto un proceso, como p. ej. llanto, lloriqueo, suspiro, aullido, etc. Ejemplos afines los encontramos en otras CU españolas cuyos sustantivos son de naturaleza deverbal como entre sollozos que sería sinónimo, en función del contexto, de sollozando. Por tanto, los sustantivos llanto, lágrima, suspiro denotan semánticamente el desarrollo del evento y sintácticamente ponen de manifiesto una estructura argumental. A la vista del ejemplo (3), la preposición unter deja de convertirse en mero nexo funcional, al mantener un estrecho vínculo con el sustantivo Träne.

\subsection{Fijación externa e interna [X entre lágrimas $X]$ y \\ [X unter Tränen $X$ ]; [entre X lágrimas $X]$ y [unter $X$ Tränen]}

De acuerdo con la combinatoria o fijación externa, se observa en ambas CU una clara preferencia por colocados verbales que son verbos de comunicación o verba dicendi. Al tratarse de una CU en la que el componente emocional (de estado) está presente, hay que destacar el hecho de que las CU entre lágrimas y unter Tränen coocurran con verbos en los que se evidencien relaciones sociales o emociones.

La Tabla 1 proporciona una lista de los 25 primeros colocados más frecuentes que aparecen inmediatamente a la izquierda y a la derecha de la CU unter Tränen a una distancia de 5 palabras, tanto sustantivos como verbales estos últimos conjugados en diferentes formas verbales. Los datos sugieren que los verbos que aparecen en el top según el índice $\log$ Dice, el índice estadístico desde un enfoque lexicográfico más fiable, lo conforman verbos directivos o exhortativos tales como bitten y flehen que tienen una alta frecuencia de aparición. En español no hemos detectado verbos exhortativos equivalentes del verbo flehen, anflehen (suplicar, implorar). La combinatoria de los colocados ver- 
bales nos muestra, entre otros muchos aspectos, qué contextos pueden predominar. Por lo que atañe a la situación de uso de los verbos flehen y bitten en combinación con unter Tränen se observa una clara tendencia del registro de habla elevado propio de contextos literarios o religiosos:

(4) Turribius erschrak sehr, als er davon hörte, er weigerte sich, diese Würde anzunehmen und flehte unter Tränen zu Gott, ihm diese Last weg zu nehmen. [http://www.heiligenlegenden.de/monate/ maerz/23/turribius/home.html]

\section{Candidatos de colocaciones}

\begin{tabular}{|c|c|c|c|c|c|}
\hline \multirow[t]{2}{*}{ Página 1} & \multicolumn{5}{|l|}{ Siguiente > } \\
\hline & $\frac{\text { Cooccurrence }}{\text { count }}$ & $\frac{\text { Candidate }}{\text { count }}$ & $\underline{\text { T-score }}$ & $\underline{\text { MI }}$ & $\underline{\text { logDice }}$ \\
\hline$\underline{P} \mid \underline{N}$ flehte & 64 & 9,026 & 7.999 & 13.786 & 6.784 \\
\hline$\underline{P} \mid \underline{N}$ Myrtha & 26 & 1,024 & 5.099 & 15.626 & 6.272 \\
\hline$\underline{P} \mid \underline{N}$ gestand & 111 & 38,601 & 10.534 & 12.484 & 6.226 \\
\hline$\underline{P} \mid \underline{N}$ beichtet & 25 & 3,537 & 5.000 & 13.781 & 5.919 \\
\hline$\underline{P} \mid \underline{N}$ gesteht & 108 & 51,582 & 10.390 & 12.026 & 5.845 \\
\hline$\underline{P} \mid \underline{N}$ Schluchzen & 27 & 7,203 & 5.195 & 12.866 & 5.685 \\
\hline$\underline{P} \mid \underline{N}$ angefleht & 19 & 3,092 & 4.359 & 13.579 & 5.572 \\
\hline$\underline{P} \mid \underline{N}$ schluchzte & 25 & 8,298 & 4.999 & 12.551 & 5.484 \\
\hline$\underline{\mathrm{P}} \mid \underline{\mathrm{N}}$ beteuerte & 29 & 11,278 & 5.384 & 12.322 & 5.481 \\
\hline$\underline{P} \mid \underline{N}$ fleht & 22 & 6,231 & 4.690 & 12.780 & 5.473 \\
\hline$\underline{P} \mid \underline{N}$ gebeichtet & 18 & 4,391 & 4.242 & 12.995 & 5.357 \\
\hline$\underline{P} \mid \underline{N}$ Rührung & 27 & 12,013 & 5.195 & 12.128 & 5.329 \\
\hline$\underline{P} \mid \underline{N}$ umarmten & 19 & 5,924 & 4.358 & 12.641 & 5.289 \\
\hline$\underline{P} \mid \underline{N}$ Seufzern & 13 & 1,316 & 3.605 & 14.264 & 5.234 \\
\hline$\underline{\mathrm{P}} \mid \underline{\mathrm{N}}$ stammelte & 23 & 10,175 & 4.795 & 12.136 & 5.223 \\
\hline$\underline{\underline{P}} \mid \underline{N}$ umarmte & 35 & 21,261 & 5.914 & 11.679 & 5.197 \\
\hline$\underline{\mathrm{P}} \mid \underline{\mathrm{N}}$ Nayirah & 11 & 87 & 3.317 & 17.942 & 5.159 \\
\hline$\underline{p} \mid \underline{N}$ Starken & 33 & 21,927 & 5.743 & 11.550 & 5.082 \\
\hline$\underline{P} \mid \underline{N}$ Verzeihung & 38 & 28,746 & 6.162 & 11.363 & 5.006 \\
\hline$\underline{P} \mid \underline{N}$ stockend & 14 & 4,760 & 3.741 & 12.516 & 4.958 \\
\hline$\underline{\mathrm{P}} \mid \underline{\mathrm{N}}$ beichtete & 11 & 2,129 & 3.316 & 13.329 & 4.893 \\
\hline$\underline{p} \mid \underline{N}$ Bluttränen & 9 & 273 & 3.000 & 16.003 & 4.843 \\
\hline$\underline{P} \mid \underline{N}$ ermahnen & 18 & 10,745 & 4.241 & 11.704 & 4.829 \\
\hline$\underline{P} \mid \underline{N}$ schluchzend & 12 & 4,202 & 3.463 & 12.474 & 4.791 \\
\hline P $\mid \bar{N}$ betete & 29 & 25,134 & 5.383 & 11.166 & 4.757 \\
\hline
\end{tabular}

Tabla 1. Ejemplo de colocados de la CU unter Tränen

Del ejemplo (4) se desprende que el sujeto paciente de la acción es Gott (zu X $\mathrm{X}_{\text {Gott/Hern/Jesus }}$ flehen). 
Los verba dicendi conforman un grupo igualmente numeroso, sobre todo en lo que concierne a verbos en alemán como beichten, berichten, sich entschuldigen, erzählen o gestehen. Este último es el más significativo del grupo por frecuencia de coaparición y mantiene un significado afín con la CU unter Tränen por el hecho de que reconocer una culpa (gestehen) encierra un momento de una gran carga emocional (unter Tränen).

\section{Candidatos de colocaciones}

\begin{tabular}{|c|c|c|c|c|c|}
\hline \multirow[t]{2}{*}{ Página 1} & \multicolumn{5}{|l|}{ Siguiente > } \\
\hline & $\frac{\text { Cooccurrence }}{\underline{\text { count }}}$ & $\frac{\text { Candidate }}{\text { count }}$ & $\underline{\text { T-score }}$ & $\underline{\mathrm{MI}}$ & $\underline{\text { logDice }}$ \\
\hline$\underline{P} \mid \underline{N}$ sollozos & 10 & 779 & 3.162 & 15.377 & 7.782 \\
\hline$\underline{P} \mid \underline{N}$ susurró & 4 & 735 & 2.000 & 14.139 & 6.503 \\
\hline$\underline{P} \mid \underline{N}$ Roqué & 3 & 629 & 1.732 & 13.949 & 6.198 \\
\hline$\underline{\mathrm{P}} \mid \underline{\mathrm{N}}$ despidió & 11 & 4,863 & 3.316 & 12.873 & 6.015 \\
\hline$\underline{P} \mid \underline{N}$ abrazan & 4 & 1,360 & 2.000 & 13.252 & 5.985 \\
\hline$\underline{\mathrm{P}} \mid \underline{\mathrm{N}}$ escoltado & 3 & 1,230 & 1.732 & 12.981 & 5.663 \\
\hline$\underline{P} \mid \underline{N}$ abrazos & 10 & 7,643 & 3.162 & 12.083 & 5.294 \\
\hline$\underline{\mathrm{P}} \mid \underline{\mathrm{N}}$ lamentos & 3 & 2,030 & 1.732 & 12.259 & 5.165 \\
\hline$\underline{P} \mid \underline{N}$ repetía & 4 & 3,144 & 2.000 & 12.043 & 5.088 \\
\hline$\underline{\mathrm{P}} \mid \underline{\mathrm{N}}$ emocionado & 9 & 8,066 & 2.999 & 11.853 & 5.071 \\
\hline$\underline{P} \mid \underline{N}$ despedirse & 4 & 4,154 & 1.999 & 11.641 & 4.752 \\
\hline$\underline{P} \mid \underline{N}$ viudas & 3 & 3,375 & 1.731 & 11.525 & 4.589 \\
\hline$\underline{P} \mid \underline{N}$ adiós & 10 & 13,279 & 3.161 & 11.286 & 4.550 \\
\hline$\underline{P} \mid \underline{N}$ sonrisas & 5 & 7,292 & 2.235 & 11.151 & 4.356 \\
\hline$\underline{\mathrm{P}} \mid \underline{\mathrm{N}}$ despedido & 5 & 7,753 & 2.235 & 11.062 & 4.275 \\
\hline$\underline{\mathrm{P}} \mid \underline{\mathrm{N}}$ despedida & 9 & 18,041 & 2.998 & 10.692 & 3.975 \\
\hline$\underline{P} \mid \underline{N}$ confiesa & 5 & 11,693 & 2.234 & 10.469 & 3.724 \\
\hline$\underline{P} \mid \underline{N}$ emoción & 15 & 37,968 & 3.870 & 10.355 & 3.668 \\
\hline$\underline{\mathrm{P}} \mid \underline{\mathrm{N}}$ recorren & 3 & 7,796 & 1.731 & 10.317 & 3.531 \\
\hline$\underline{P} \mid \underline{N}$ gritos & 7 & 19,523 & 2.644 & 10.215 & 3.503 \\
\hline$\underline{\mathrm{P}} \mid \underline{\mathrm{N}}$ retiró & 3 & 8,347 & 1.731 & 10.219 & 3.440 \\
\hline$\underline{P} \mid \underline{N}$ confesó & 3 & 8,655 & 1.731 & 10.167 & 3.392 \\
\hline$\underline{P} \mid \underline{N}$ rostros & 4 & 12,445 & 1.998 & 10.058 & 3.317 \\
\hline$\underline{P} \mid \underline{N}$ agradeció & 4 & 12,647 & 1.998 & 10.034 & 3.295 \\
\hline$\underline{P} \mid \underline{N}$ abandonó & 4 & 14,012 & 1.998 & 9.887 & 3.154 \\
\hline$\underline{\mathrm{P}} \mid \underline{\mathrm{N}}$ impotencia & 3 & 10,377 & 1.730 & 9.905 & 3.148 \\
\hline$\underline{\mathrm{P}} \mid \underline{\mathrm{N}}$ rabia & 5 & 18,957 & 2.234 & 9.772 & 3.058 \\
\hline$\underline{P} \mid \underline{N}$ desesperación & 3 & 11,290 & 1.730 & 9.783 & 3.034 \\
\hline$\underline{\mathrm{P}} \mid \underline{\mathrm{N}}$ aplausos & 3 & 11,471 & 1.730 & 9.760 & 3.013 \\
\hline$\underline{\mathrm{P}} \mid \underline{\mathrm{N}}$ risas & 5 & 19,683 & 2.233 & 9.718 & 3.006 \\
\hline$\underline{P} \mid \underline{N}$ pidió & 9 & 42,302 & 2.996 & 9.462 & 2.777 \\
\hline
\end{tabular}

Tabla 2. Ejemplo de colocados de la CU “entre lágrimas” 
Tal y como se desprende de la tabla 2, los casos más representativos lo componen verba dicendi (repetir, confesar, pedir), verbos de interacción social (agradecer) y verbos de contacto (abrazar). Se constata en ambos idiomas un grupo recurrente de verbos que expresan relaciones sociales como sich verabschieden, Abschied nehmen y despedirse o decir adiós en español en los que se describe mayoritariamente el cese de la actividad dentro de un contexto deportivo:

(5) Dabei verabschiedete er sich unter Tränen von den fast 24.000 Zuschauern im Arthur Ashe Stadium in New York. [http://www. whoswho.de/bio/andre-agassi.html]

(6) pero que sirvió como perfecto colofón a la carrera de Joseba Etxeberría, quién se despidió de San Mamés entre lágrimas con más de 400 partidos como bagaje. [http://agendapolitica.es/deportes/elgetafe-regresa-a-europa-por-la-puerta-grande.html]

En español y en alemán se observan diferencias de uso con relación al colocado verbal sich umarmen y abrazar. En español, se enuncia con frecuencia en presente en 3. ${ }^{a}$ persona del plural con sentido recíproco, y en cambio en alemán se acentúa más el uso en pasado igualmente en 3 . $^{\mathrm{a}}$ persona del plural. Los lemas y sus correspondientes formas verbales evidencian en el corpus distintas estrategias de implicación del interlocutor. En el caso del presente de indicativo en 3. ${ }^{\mathrm{a}}$ personal del plural (sie umarmen sich, se abrazan), los lemas verbales utilizados poseen una función descriptiva.

Antes de pasar a la fijación interna, exponemos brevemente la expansión a la derecha de las CU [unter Tränen X] y [entre lágrimas X]. El análisis de las listas de colocados a la derecha de las CU presenta elementos interesantes (unter Tränen des/der/und y entre lágrimas dely).

La expansión de [unter Tränen $N P_{\text {genitivo }}$ que actúa como modificador del lexema 'Träne' engloba sustantivos que pertenecen mayoritariamente al ámbito de las emociones (Rührung, Mitleid). En cuanto a la secuencia española [entre lágrimas $N P_{\text {genitivo }}$ se constatan sustantivos de diferente naturaleza semántica (rabia, tristeza, emoción). El análisis de los corpus nos ha permitido constatar que especialmente algunos colocados sustantivos muestran una marcada tendencia a la expansión a la derecha entre lágrimas y sollozos, entre lágrimas y abrazos, entre lágrimas y aplausos. En alemán los sustantivos más prototípicos refuerzan el componente emocional de llorar por ser sinónimos de Trä- 
nen (unter Tränen und Bluttränen, unter Tränen und Schluchzen, unter Tränen und Seufzern). De ahí que algunos de estos binomios presenten un cierto grado de lexicalización (Mellado Blanco, 2015).

(7) Wenn die Kinder dieser Welt meine Worte nicht annehmen und verachten - die ich unter Tränen und Bluttränen laut herausschreie - weiterhin den Vergnügungen und dem Müßiggang frönen, wird der Verfall wie ein Dieb in der Nacht hereinbrechen. [http://www. kommherrjesus.de/index.php]

Asimismo, tenemos que tener en cuenta que las lenguas difieren en su morfología, así, por ejemplo, en alemán, donde la composición es mucho más frecuente que en español, la equivalencia de lágrimas de emoción o lágrimas de cocodrilo es un compuesto que conforma una unidad ortográfica (Gefühlstränen, Kokrodilstränen) en parte por el hecho de que el léxico alemán es más proclive a un mayor número de compuestos de este tipo frente al español, que presenta una expansión hacia la derecha en forma de complemento del nombre (lágrimas de $\mathrm{N}_{\text {emoción/(cocodrilo/alegria }}$. La diferencia estriba en la morfología léxica de cada lengua, concretamente en la preferencia del alemán por palabras compuestas que conforman una unidad ortográfica (Lachtränen) frente a las formaciones compuestas en español que no están unidas gráficamente (lágrimas de emoción). Este rasgo es fundamental cuando manejamos corpus y consultamos frecuencias de aparición de grupos de palabras, que más adelante comprobaremos en relación a la fijación interna.

En alemán, hemos observado que la palabra compuesta Kokrodilstränen como CU se emplea tanto con la preposición mit como con la preposición unter (unter Kokrodilstränen, mit Kokrodilstränen). Curiosamente, en español no aparece ningún caso con la preposición entre lágrimas de cocodrilo, únicamente con la preposición con. Esta CU con expansión a la derecha se usa frecuentemente en oraciones de imperativo negativo (no me vengas con lágrimas de cocodrilo) o en oraciones afirmativas:

(8) Lo que me parece patética es la actitud de algunos, que vienen con lágrimas de cocodrilo diciendo que se están cargando el ciclismo, sois vosotros los que os lo habéis cargado, pandilla de golfos y maleantes, iros a llorar con vuestra p... madre. [http://blogs.abc.es/] 
El caso de lágrimas de risa es frecuente en alemán acompañado de la preposición entre (unter Lachtränen) frente al español que se decanta por la combinación con la preposición con lágrimas de risa. En el corpus solo hemos encontrado un caso (entre lágrimas de risa).

En relación con la fijación interna [unter $+\mathrm{X}+$ Tränen] y [entre $+\mathrm{X}$ + lágrimas] el análisis de los corpus constata que la expansión interna en español es menor que en alemán, la tendencia del adjetivo a la posición posnominal promueve secuencias del tipo lágrimas amargas. En este punto, consideramos fundamental hacer hincapié en el fenómeno de la posición de los adjetivos en ambas lenguas. La fuerza de la posición atributiva de los adjetivos en alemán es mucho mayor que en español porque se da el caso en español de que el significado según sea la posición del adjetivo cambia (un pobre hombre frente a un hombre pobre). Seco (1975: 5) señala que "el sentido recto siempre se conserva en el adjetivo pospuesto, mientras que el antepuesto está más o menos deformado". Este pequeño inciso nos ayuda a entender que la fijación interna sea, por regla general, en secuencias del tipo [PREP $+\mathrm{X}+\mathrm{S}$ ] mayor en alemán que en español. Una de las varas de medir la mayor o menor fijación de las CU en el discurso radica en observar el comportamiento de este tipo de secuencias en su fijación interna. Cuanto mayor sea el nivel de saturación de los slots internos, menor será la fijación y por tanto más bajo será su nivel de lexicalización. Sirva como ejemplo de repente cuyo slot interno $\mathrm{X}$ [de $\mathrm{X}$ repente], a la luz de los corpus consultados, es prácticamente nulo.

Entre los slots adjetivos más recurrentes en alemán cabe destacar adjetivos que inciden en la intensidad del llanto (unter vielen Tränen), o establecen una relación cognitiva con el concepto del agua (unter strömenden Tränen) o con sensaciones gustativas (unter bitteren Tränen). Un ejemplo que se asemeja al que hemos señalado más arriba unter Tränen und Bluttränen posee de nuevo un valor intensificador del acto de llorar (unter blutigen Tränen) que hace acto de presencia mayoritariamente en contextos literarios y adquiere un claro significado translaticio:

(9) Die Wirtin wurde immer aufmerksamer, als er aber daran kam, wie er die schöne Jungfrau aus dem Loche erlöst und sich mit ihr verlobt hatte, da schloss sie ihn in ihre Arme und rief unter blutigen Tränen: [http://internet-maerchen.de/] 
La CU unter heißen Tränen está presente en registros elevados junto con verbos en tiempos pasados y al igual que unter blutigen Tränen existe una traslación metafórica:

\section{Füller-Tabelle \\ Füller zum Suchmuster "unter|Unter \# Tränen", Feld 3 \\ 126 unterschiedliche Füller}

\begin{tabular}{|c|c|c|c|c|c|}
\hline Lückenfüller & Anzahl & Prozentanteil & Tag & Kommentar & Sortierung \\
\hline vielen & 217 & 32,63 & & & 0 \\
\hline den & 80 & 12,03 & & & 0 \\
\hline heißen & 50 & 7,52 & & & 0 \\
\hline bitteren & 32 & 4,81 & & & 0 \\
\hline strömenden & 28 & 4,21 & & & 0 \\
\hline ihren & 22 & 3,31 & & & 0 \\
\hline tausend & 12 & 1,80 & & & 0 \\
\hline viel & 11 & 1,65 & & & 0 \\
\hline schmerzlichen & 10 & 1,50 & & & 0 \\
\hline meinen & 9 & 1,35 & & & 0 \\
\hline blutigen & 7 & 1,05 & & & 0 \\
\hline einigen & 7 & 1,05 & & & 0 \\
\hline großen & 6 & 0,90 & & & 0 \\
\hline heftigen & 6 & 0,90 & & & 0 \\
\hline häufigen & 6 & 0,90 & & & 0 \\
\hline dicken & 5 & 0,75 & & & 0 \\
\hline die & 5 & 0,75 & & & 0 \\
\hline diesen & 5 & 0,75 & & & 0 \\
\hline erneuten & 5 & 0,75 & & & 0 \\
\hline reichlich & 5 & 0,75 & & & 0 \\
\hline stillen & 5 & 0,75 & & & 0 \\
\hline bittern & 4 & 0,60 & & & 0 \\
\hline seinen & 4 & 0,60 & & & 0 \\
\hline Tränen & 3 & 0,45 & & & 0 \\
\hline falschen & 3 & 0,45 & & & 0 \\
\hline schmerzvollen & 3 & 0,45 & & & 0 \\
\hline stummen & 3 & 0,45 & & & 0 \\
\hline ständigen & 3 & 0,45 & & & 0 \\
\hline zahlreichen & 3 & 0,45 & & & 0 \\
\hline Umstände & 2 & 0,30 & & & 0 \\
\hline fließenden & 2 & 0,30 & & & 0 \\
\hline hellen & 2 & 0,30 & & & 0 \\
\hline hervorbrechenden & 2 & 0,30 & & & 0 \\
\hline hervorquellenden & 2 & 0,30 & & & 0 \\
\hline laufenden & 2 & 0,30 & & & 0 \\
\hline unendlichen & 2 & 0,30 & & & 0 \\
\hline weiteren & 2 & 0,30 & & & 0 \\
\hline zornigen & 2 & 0,30 & & & 0 \\
\hline
\end{tabular}

Tabla 3. Ejemplos de slots internos de la CU "unter X Tränen"

La mayoría de los slots internos de la CU unter $X$ Tränen actúan como valor intensificador del lexema "Träne" (unter heißen/bitteren/ 
strömenden/heftigen Tränen). En español se evidencia una saturación mínima de los slots internos (adjetivos) de la CU entre X lágrimas. La explicación de este fenómeno guarda relación con la equivalencia de entre lágrimas con el gerundio llorando, por ello en el corpus apenas se detectan slots internos con valor modal y temporal. Cuando se intercala un determinante (las, mis, tus) la preposición expresa un significado local o divisivo:

(10) Pero el dios sigue susurrando entre las lágrimas. [http://avisos. realbiblioteca.es $/ \mathrm{p}=$ article\&aviso $=43 \&$ art $=879 \&$ lang $=\mathrm{es}]$

Como se desprende del ejemplo (10), los determinantes desempeñan un papel fundamental para anular el significado idiomático o fraseológico de entre lágrimas. Los valores modal y temporal dejan paso al valor local o divisivo o a verbos con régimen verbal o complemento preposicional (escoger entre las lágrimas o la risa). Cuando los slots son varios, el español exhibe sustantivos acompañados de una conjunción (entre sonrisas y lágrimas, entre aplausos y lágrimas, entre risas y lágrimas, entre un mar de lágrimas). En buena parte de los casos se observa una antítesis o contraste entre el par de sustantivos (sonrisa y lágrima, risa y lágrima) que mitigan el efecto o el sentimiento negativo del llanto (lágrima).

\section{Reflexiones finales}

Los corpus brindan a los lingüistas la posibilidad de manejar datos voluminosos que se desmarcan de los datos que proceden del juicio intuitivo e introspectivo del lingüista y que están insertos en contextos discursivos reales. Ahora bien, es preciso puntualizar que trabajar con corpus no está exento de dificultades. Una de las limitaciones de nuestro estudio consiste en que los textos del corpus que hemos manejado proceden mayoritariamente del lenguaje periodístico. Otra limitación ha sido la dificultad para obtener información de uso a nivel nivel supraoracional, hecho que dificulta distinguir aspectos del contexto que aportan información valiosa para el cotexto inmediato de las CU objeto de estudio. Con todo y con ello, es evidente que los corpus lingüísticos aportan datos reveladores (estadísticos) que proceden de evidencias 
empíricas y favorecen un análisis más exhaustivo en el campo de la fraseología.

De acuerdo con la combinatoria o fijación externa de las CU entre lágrimas y unter Tränen, se observa una clara preferencia por colocados verbales que son verba dicendi y de contacto. En español entre lágrimas va muy ligado a la forma de gerundio del verbo llorar llorando, y en alemán se suple esta carencia exhibiendo el valor modal. Asimismo, es llamativo el fuerte componente emocional y expresivo del significado pragmático de los binomios entre lágrimas y sollozos y unter Tränen und Bluttränen. Por último, en relación a los slots (X) en la fijación interna [entre X lágrimas] y [unter X Tränen] el hecho de que en español el adjetivo tienda a la posición posnominal implica un nivel menor de saturación del slot X frente al alemán (unter bitteren Tränen - entre lágrimas amargas).

\section{Bibliografía}

Burger, Harald. 2010. Phraseologie: eine Einführung am Beispiel des Deutschen. Berlin: Erich Schmidt Verlag.

Cabezas Holgado, Emilio. 2013. La predicación: Las construcciones en abanico. Aplicaciones al español. http:// eprints.ucm.es/22365/1/T34644. pdf [Acceso 27/03/2017].

Dobrovol'skij, Dmitrij. 2011. Phraseologie und Konstruktionsgrammatik. En Lasch, Alexander \& Ziem, Alexander (ed.) Konstruktionsgrammatik III. Aktuelle Fragen und Lösungsansätze. Tübingen: Stauffenburg, 111130.

Fleischer, Wolfgang. 1997. Phraseologie der deutschen Gegenwartssprache. Tübingen: De Gruyter.

Häusermann, Jürg. 1977. Phraseologie. Hauptprobleme der deutschen Phraseologie auf der Basis sowjetischer Forschungsergebnisse. (=Linguistische Arbeiten 47). Tübingen: Max Niemeyer.

Helbig, Gerhard \& Buscha, Joachim. 1996. Deutsche Grammatik. Ein Handbuch für den Ausländerunterricht. Leipzig: Langenscheidt Verlag Enzyklopädie.

Kiss, Tibor; Müller, Antje; Roch, Claudia; Stadtfeld, Tobias; Börner, Katharina \& Duzyein, Monika (ed.). 2014. Handbuch für die Bestimmung und Annotation von Präpositionsbedeutungen im Deutschen (Bochumer Linguistische Arbeiten 14). Bochum: Sprachwissenschaftliches Institut, Ruhr-Universität Bochum. 
Martínez García, Hortensia. 2012. Viejos y nuevos valores de las preposiciones españolas. Verba 39: 7-34.

Mellado Blanco, Carmen. 2015. Phrasem-Konstruktionen und lexikalische Idiom Varianten: der Fall der komparativen Phraseme des Deutschen. En Engelberg, Stefan; Meliss, Meike; Proost, Kristel \& Winkler, Edeltraud (ed.) Argumentstruktur - Valenz - Konstruktionen. Tübingen: Narr, 217-235.

Mellado Blanco, Carmen \& Belén López Meirama. Esquemas sintácticos de preposición + sustantivo: el caso de [entre + Splural/corporal]. En Mellado Blanco, Carmen; Berty, Katrin \& Olza, Inés (ed.) Discurso repetido y fraseología textual (español y español-alemán). Frankfurt am Main: Vervuert / Iberoamericana (en prensa).

Pérez Hernández, Chantal \& Moreno Ortiz, Antonio. 2009. Lingüística Computacional y Lingüística de Corpus. En Rodríguez Ortega, Nuria (ed.) Teoría y literatura artística en la sociedad digital: construcción y aplicabilidad de colecciones textuales informatizadas. Gijón: Trea, 67-96.

Sánchez, Aquilino \& Almela, Moisés. 2010. A Mosaic of Corpus Linguistics. Frankfurt am Main: Peter Lang.

Seco, Manuel. 1975. Manual de gramática española. Madrid: Alfaguara.

Sinclair, John. 1991. Corpus, Concordance, Collocation. Oxford: Oxford University Press.

Steyer, Kathrin. 2013. Usuelle Wortverbindungen. Zentrale Muster des Sprachgebrauchs aus korpusanalytischer Sicht. Tübingen: Narr.

Wittgenstein, Ludwig. 1953. Philosophische Untersuchungen. Oxford: Blackwell. 